\title{
ACE INHIBITORS AND AT1R BLOCKERS FOR COVID-2019: FRIENDS OR FOES ?
}

Focosi Daniele ${ }^{1, \#}$

Tuccori Marco ${ }^{2}$

Maggi Fabrizio ${ }^{2}$

${ }^{1}$ North-Western Tuscany Blood Bank, Pisa University Hospital.

${ }^{2}$ Unit of Adverse Drug Reactions Monitoring, Pisa University Hospital.

${ }^{3}$ Division of Virology, University of Pisa.

\#Corresponding author : daniele.focosi@gmail.com . via Paradisa 2, 56124 Pisa, Italy. Phone : +39 050 996541.

Word count : 1849.

Abbreviations : COVID-19 : coronavirus disease 2019; ACEI : ACE inhibitors; ARB : angiotensin receptor blockers; SARS: severe acute respiratory syndrome; ALI : acute lung injury ; ARDS : acute respiratory distress syndrome.

\begin{abstract}
Human respiratory beta coronavirus are emerging causes for Public Health Emergencies of International Concern (PHEIC). SARS-CoV2 is circulating worldwide since November 2019. We review here the cardiovascular morbidity and mortality in COVID-19, and data supporting the role for dysregulation of the RAS counterregulatory axis due to binding of SARS-CoV2 S protein to ACE2 receptor. Since this counterregulatory axis provides benefits not only on the cardiovascular front but also in acute lung injury, we speculate on potential use of ACE inhibitors and $A T_{1} R$ blockers in critically ill COVID-19 patients, and report current evidences.
\end{abstract}




\section{Human respiratory betacoronaviruses}

Three respiratory betacoronaviruses of bat origin and various intermediate hosts have emerged in humans in China and Middle East in the last two decades, rapidly evolving into Public Health Emergencies of International Concern (PHEIC). Severe acute respiratory syndrome (SARS) was first identified in Guangdong, China in November 2002 and spread to 8096 cases in 29 countries for an overall case fatality rate (CFR) of 9.6\% before WHO declared worldwide containment in July 2003, despite a few new cases in 2004 . The responsible betacoronavirus was named SARS-CoV. In September 2012 the cause for the Middle-Eastern Respiratory syndrome (MERS) was identified in the MERS-CoV, and the epidemic has to date caused approximatively 2500 cases with a CFR of 34\%. In January 2020, following an outbreak of pneumonia in Wuhan, Chinese scientists isolated a novel betacoronavirus, which was initially named 2019-nCoV[1] and then renamed SARS-CoV2 by the International Committee on Taxonomy of Viruses (ICTV). The nosological entity was named COVID-2019[2], and the epidemic is currently ongoing worldwide.

\section{Cardiovascular morbidity and mortality in COVID-19}

COVID-19 has a far lower estimated mortality than SARS but still high morbidity, with approximatively $10 \%$ of patients requiring ICU staying: up to $50 \%$ of hospitalized patients have a chronic medical illness, $80 \%$ of which are cardiovascular or cerebrovascular. Overall the CFR remains low at $2.3 \%$, but jumps to $6 \%$ in hypertensives and $10.5 \%$ in patients with cardiovascular disease[3].

Table 1. Evidences for cardiovascular damage from published COVID-19 case series.

\begin{tabular}{|l|l|l|}
\hline \multicolumn{1}{|c|}{ Reference } & $\begin{array}{c}|c| \\
\text { (confirmed } \\
\text { cases) }\end{array}$ & \\
\hline $\begin{array}{l}\text { Huang C et al, Lancet } \\
\text { 2020, [4] }\end{array}$ & 41 & $\begin{array}{l}5 \text { cases (12\%) showed increased high-sensitivity cardiac troponin I } \\
\text { and received a diagnosis of virus-related acute cardiac injury. }\end{array}$ \\
\hline $\begin{array}{l}\text { Chen N et al, Lancet } \\
\text { 2020, [5] }\end{array}$ & 99 & $40 \%$ had pre-existing cardiovascular or cerebrovascular disease. \\
\hline
\end{tabular}




\begin{tabular}{|c|c|c|}
\hline $\begin{array}{l}\text { Xiao-Wei X et al., BMJ } \\
2020[6]\end{array}$ & 62 & $\begin{array}{l}5(8 \%) \text { with pre-existing hypertension and } 1 \text { with cerebrovascular } \\
\text { disease. At admission, mean arterial pressure } 97 \mathrm{~mm} \mathrm{Hg} \mathrm{(87-106).}\end{array}$ \\
\hline $\begin{array}{l}\text { Wang D et al., JAMA } \\
\text { 2020[7] }\end{array}$ & 138 & $\begin{array}{l}43(32.1 \%) \text { with pre-existing hypertension, } 7 \text { (5.1\%) with pre- } \\
\text { existing unspecified CV disease, and } 7 \text { (5.1) with pre-existing } \\
\text { cerebrovascular disease. At admission median arterial pressure } 90 \\
\text { mm Hg, normal values of mean high-sensitivity cardiac troponin I. } \\
\text { The probability of being admitted to ICU was higher in those with } \\
\text { hypertension and CV disease. Acute cardiac injury was a } \\
\text { complication in } 10 \text { patients (7.2\%) and shock in } 12 \text { patients }(8.7 \%)\end{array}$ \\
\hline $\begin{array}{l}\text { Yang X et al, Lancet } \\
2020[8]\end{array}$ & $\begin{array}{l}710(52 \\
\text { critically } \\
\text { ill) }\end{array}$ & $\begin{array}{l}32 \text { died - pre-existing chronic cardiac disease } 5 \text { (10\%) Compared } \\
\text { with survivors, non-survivors were older ( } 65 \text { vs } 52 \text { years), more } \\
\text { likely to develop ARDS ( } 81 \% \text { vs } 45 \%) \text {, and more likely to receive } \\
\text { mechanical ventilation ( } 94 \% \text { patients vs } 35 \% \text { ), either invasively or } \\
\text { non-invasively. } 23 \% \text { had cardiac injury. Mean systolic blood } \\
\text { pressure was } 133 \text { mmHg in survivors vs } 140 \text { in non survivors }\end{array}$ \\
\hline $\begin{array}{l}\text { Wu Z et al, JAMA } 2020 \\
\text { [9] }\end{array}$ & 44,672 & $\begin{array}{l}\text { CFR was elevated among those with preexisting comorbid } \\
\text { conditions }-10.5 \% \text { for cardiovascular disease, } 7.3 \% \text { for diabetes, } \\
6.3 \% \text { for chronic respiratory disease, } 6.0 \% \text { for hypertension }\end{array}$ \\
\hline
\end{tabular}

Viral illness is a well-known destabilizing factor in chronic cardiovascular disease: e.g. it can destabilize coronary plaques through several mechanisms including systemic inflammatory responses, but we will focus here on an additional mechanism of action, namely ACE2 axis dysregulation.

\section{ACE2 as a viral receptor and its implications in metabolism}

The human receptor for the viral Spike (S) protein S1 subunit of SARS-CoV was identified in the catalytic domain of angiotensin-converting enzyme II (ACE2) [10], although vimentin-S protein interaction is also required for cell entry[11]. In 2005 ACE2 was also identified as the receptor for the other less invasive alphacoronavirus HCoV-NL63[12], albeit with far lower affinity. Similarly, Zhou et al identified ACE2 as the cell receptor of SARS-CoV2 [13], with higher affinity than SARS-CoV [14, 15]. Single-cell RNA sequencing (scRNA-Seq) technique and single-cell transcriptomes identified high ACE2 expression in apical surface of type II alveolar cells (AT2) of lung [16-19], esophagus upper and stratified epithelial cells, absorptive enterocytes from ileum and colon[18], cholangiocytes [20], myocardial cells, endothelium of coronary and 
intrarenal vessels [21], kidney proximal tubule cells, bladder urothelial cells[16], and tongue epithelium [22].

ACE2 is a type I transmembrane protein, with an extracellular $N$-terminal domain containing the active terminal monocarboxypeptidase site (that degrades Ang I into a Ang $1-9$ and Ang II into Ang $1-7$ ) and a short

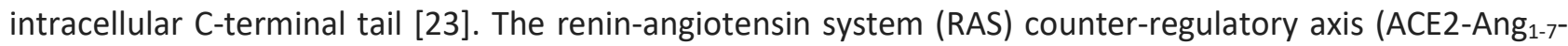
Mas receptor) opposes the classical arm (ACE-Ang II-AT 1 receptor) actions, presenting anti-inflammatory, anti-oxidative, and anti-fibrotic effects. Ang 1-7 $_{1}$ is a biologically active peptide exerting a wide array of actions, many of which are opposite to those attributed to Ang II. Taken together, the cardioprotective

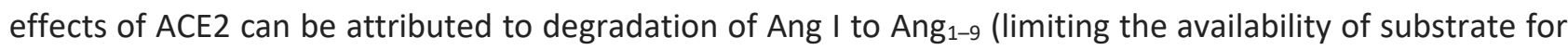
ACE action), degradation of Ang II (limiting its detrimental effects), and generation of Ang $1-7$ (exerting its cardioprotective effects) [24].

S protein from SARS-CoV, but not HNL63-CoV, induces TNF- $\alpha$-converting enzyme (TACE)/ADAM17dependent shedding of the ACE2 ectodomain $[25,26]$, which is inhibited by calmodulin under physiological circumstances[27]. The TACE inhibitor TAPI-2 blocks both the SARS-CoV S protein-induced shedding of ACE2, and TNF- $\alpha$ production in lung tissues [28]. TMPRSS2 was found to compete with the metalloprotease ADAM17 for ACE2 processing, but only cleavage by TMPRSS2 resulted in augmented SARSS-driven entry [29]. Binding of SARS-S to ACE2 triggers subtle conformational rearrangements in SARS-S, which are believed to increase the sensitivity of the S protein to proteolytic digest at the border between the S1 and S2 subunits [30]. Cleavage of the S protein by host cell proteases is essential for viral infectivity, and the responsible enzymes constitute potential targets for intervention. Collectively, these results indicate that TMPRSS2 and potentially related proteases promote SARS-CoV entry by 2 separate mechanisms: ACE2 cleavage, which might promote viral uptake, and SARS-S cleavage, which activates the S protein for membrane fusion. Soluble ACE2 would be enzymatically active but is saturated by SARS-CoV2 S protein: despite acting as a decoy receptor, it only partially inhibits virus entry into target cells [31].

Notably, injection of SARS-CoV S protein into mice worsens acute lung failure in vivo that can be attenuated by blocking the RAS axis with $\mathrm{AT}_{1} \mathrm{R}$ blockers [32].

Intriguingly, mortality from SARS-CoV and SARS-CoV2 is significatively higher in males than females: despite lower estrogens[33] or higher smoking could be simple explanations, an alternative stays with ACE2 gene being located on X-chromosome, making an eventual recessive infection-predisposing polymorphisms [34, 35] silenced in females. Receptor polymorphisms affecting coronavirus entry has already been proven for 
MERS-CoV[36]. A number of ACE2 variants with a potential impact on protein stability have been identified. Among these, 3 missense changes, p.Asn720Asp, p.Lys26Arg, p.Gly211Arg (MAF 0.002 to 0.015), which have never been reported in the Eastern Asia population, were predicted to interfere with protein cleavage and stabilization. Rare truncating variants likely interfering with the internalization process and one missense variant, p.Trp69Cys, predicted to interfere with 2019-nCov spike protein binding were also observed.

\section{Therapeutic opportunities targeting the RAS system in COVID-19}

ACE2 is highly expressed in patients with hypertension, diabetes, and chronic obstructive lung disease, compared to control individuals[37, 38].

To date candidate drugs targeting SARS-CoV2 include small chemicals (such as the RNA-dependent RNA polymerase inhibitors remdesivir (GS-5734) and favipiravir (T-705)) and neutralizing antibodies (either monoclonal [39] or convalescent blood products [40]). Unfortunately no such candidate has been tested in phase III clinical trials yet. On the contrary of what has happened with DPP-IV inhibitors for MERS-CoV [41], no drug with previous clinical indications is available for prescription. Nevertheless, supportive therapy with immunomodulators such as the TLR9 suppressor hydroxychloroquine [42] or the anti-IL-6 antibody tocilizumab [43] can offer treatment opportunities for improving prognosis in COVID-19 patients .

Deployment of ACE inhibitors (ACEI) or angiotensin-receptor blockers (ARB) could be of benefit in order to reduce cardiovascular mortality in elderlies, provided such therapies are tailored to individual patients. Additionally, the RAS axes has also been implicated in the regulation of inflammation, proliferation and fibrosis in acute lung injury (ALI)/acute respiratory distress syndrome (ARDS), with ACE2 having protective effects in different etiologies[44], including respiratory viruses (e.g. influenzavirus $A\left(H_{5} N_{1}\right)$ [45] or $A\left(H_{7} N_{9}\right)$ [46]). Similar benefits can be achieved by direct administration of the downstream product, Ang 1-7.

The ARB losartan and olmesartan, commonly applied for reducing blood pressure in hypertensive patients, increase cardiac $[47,48]$ and renal $[49]$ ACE2 expression in animal models. Accordingly, higher urinary ACE2 levels are found in hypertensive patients treated with olmesartan[50]. Similarly, the ACE-inhibitor lisinopril increases mRNA expression of cardiac ACE2[48]. Taken together, these observations suggest that chronic $A C E$ or $A T_{1} R$ blockade results in ACE2 upregulation, which would discourage usage. However, binding of the SARS-CoV spike protein to ACE2 leads to ACE2 downregulation, which in turn results in excessive production of angiotensin by the related enzyme ACE, while less ACE2 is capable of converting it to the vasodilator heptapeptide angiotensin 1-7. This in turn contributes to lung injury, as angiotensin-stimulated 
$A T_{1} R$ results in increased pulmonary vascular permeability, thereby mediating increased lung pathology [32]. Therefore, higher ACE2 expression following chronically medicating SARS-CoV-2 infected patients with ARB, while seemingly paradoxical $[51,52]$, may protect them against acute lung injury rather than putting them at higher risk to develop SARS. Nevertheless, studies that report increases in ACE2 expression tend to involve acute injury models and/or higher doses than typically administered to patients. Data from human studies overwhelmingly imply that administration of ACEIs/ARBs does not increase ACE2 expression[53].

Accordingly, among 511 COVID-19 patients aged >65 years with hypertension, the risk of severe COVID-19 was significantly decreased $(\mathrm{OR}=0.343)$ in patients who took $A R B$ prior to hospitalization compared to patients who took no anti-hypertensive drugs [54].

In another series of 126 COVID-19 patients, However, ARBs/ACEls group had significantly lower concentrations of CRP and procalcitonin, as well as less seere disease or mortality [55].

Finally, in a multicenter retrospective series of 564 Chinese patient, hypertension without receiving ACEI/ARB therapy was an independent risk factor (odds ratio [OR], 2.07; $95 \% \mathrm{Cl}, 1.07$ to 4.00 ) for developing severe pneumonia irrespective of age [56].

In a multicenter UK trial on 53 patients, a lower rate of death or transfer to a critical care unit within 7 days in patients on an ACEI was reported compared to patients not on ACEI (OR 0.29) [57].

Discordant data have also been reported. In a case series of 274 patients from Wuhan, Patients with hypertension who had previously taken ACEI/ARB have a statistically nonsignificant tendency to develop severe pneumonia $(P=0.064)[58]$.

As suggested by other authors, this may be accounted for by two complementary mechanisms: blocking the excessive angiotensin-mediated $A T_{1} R$ activation caused by the viral infection, as well as upregulating $A C E 2$, thereby reducing angiotensin production by ACE and increasing the production of the vasodilator angiotensin 1-7[51].

Following the SARS-CoV epidemic, ACEI were suggested as SARS therapeutics $[59,60]$. Incidentally, in the context of the human immunodeficiency viruses (HIV), it has been demonstrated that higher expression levels of the HIV binding sites CCR5 and CD4 protect from, rather than increase, HIV virulence. Michel et al. reported that HIV employs its early gene Nef product for avoiding superinfection during the viral-entry step by downregulating CCR5. This Nef-mediated downregulation enhances the endocytosis rate of both CCR5 and CD4, which in turn facilitates efficient replication and spread of HIV, thereby promoting AIDS pathogenesis[61]. 
Both SARS and COVID-19 cases are normotensive at admission [62]: while SARS patients developed hypotension during staying at hospital (which is for sure a contraindication to ACEI or ARB administration), this has not been reported yet for COVID-19 patients. Whether this is due to higher affinity of S protein of SARS-CoV2 for ACE2 remains to be cleared, but at least do not pose hurdles to testing in COVID-19.

Figure 1 summarizes potential intervention points for currently approved or preclinical chemicals targeting the classical or counterregulatory RAS axes.

Of interest, a phase II clinical trial of recombinant human ACE2 (rhACE2) GSK2586881 in ARDS showed that ATII levels decreased rapidly, whereas angiotensin-(1-7) and angiotensin-(1-5) levels increased and remained elevated for 48 hours [63]. Surfactant protein D concentrations were increased, whereas there was a trend for a decrease in interleukin-6 concentrations in rhACE2-treated subjects compared with placebo. The study was unfortunately not powered to detect changes in acute physiology or clinical outcomes[63].

The low toxicity profile of ACEI and ARB encourages interventional randomized clinical trials in critically ill COVID-19 patients, escalating dose to previously treated patients and introducing ACEI to previously untreated patients [64].

We declare that we have no conflict of interest related to this manuscript. 
Figure 1. Potential intervention points in RAS axes in COVID-19. Hexagons represent chemicals that can upregulate ACE2 axis or downregulate ACE axis.
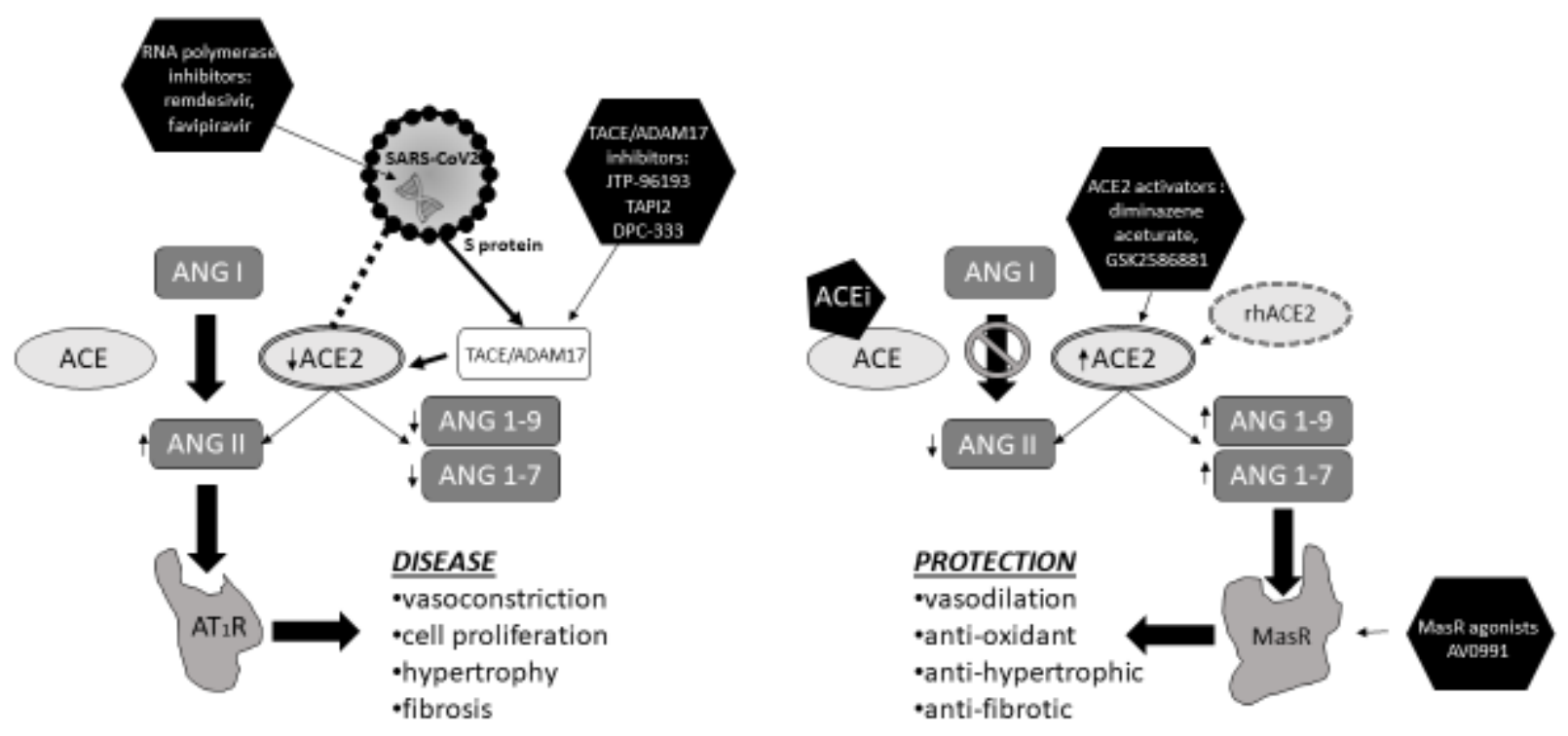
References:

1. Zhu, N., et al., A Novel Coronavirus from Patients with Pneumonia in China, 2019. New England Journal of Medicine, 2020. 382(8): p. 727-733.

2. Gorbalenya, A.E., et al., Severe acute respiratory syndrome-related coronavirus: The species and its viruses - a statement of the Coronavirus Study Group. bioRxiv, 2020: p. 2020.02.07.937862.

3. Cardiology, A.C.o., ACC Clinical Bulletin Cardiac Implications of Novel Coronavirus (COVID-19). 2020.

4. Huang, C., et al., Clinical features of patients infected with 2019 novel coronavirus in Wuhan, China. Lancet, 2020. 395(10223): p. 497-506.

5. Chen, N., et al., Epidemiological and clinical characteristics of 99 cases of 2019 novel coronavirus pneumonia in Wuhan, China: a descriptive study. Lancet, 2020. 395(10223): p. 507-513.

6. $\mathrm{Xu}, \mathrm{X} .-\mathrm{W}$. , et al., Clinical findings in a group of patients infected with the 2019 novel coronavirus (SARS-Cov-2) outside of Wuhan, China: retrospective case series. BMJ, 2020. 368: p. m606.

7. Wang, D., et al., Clinical Characteristics of 138 Hospitalized Patients With 2019 Novel CoronavirusInfected Pneumonia in Wuhan, China. Jama, 2020.

8. Yang, X., et al., Clinical course and outcomes of critically ill patients with SARS-CoV-2 pneumonia in Wuhan, China: a single-centered, retrospective, observational study. Lancet Respir Med, 2020.

9. Wu, Z. and J.M. McGoogan, Characteristics of and Important Lessons From the Coronavirus Disease 2019 (COVID-19) Outbreak in China: Summary of a Report of 72314 Cases From the Chinese Center for Disease Control and Prevention. JAMA, 2020.

10. Li, W., et al., Angiotensin-converting enzyme 2 is a functional receptor for the SARS coronavirus. Nature, 2003. 426(6965): p. 450-454.

11. Yu, Y.T., et al., Surface vimentin is critical for the cell entry of SARS-CoV. J Biomed Sci, 2016. 23: p. 14.

12. Hofmann, H., et al., Human coronavirus NL63 employs the severe acute respiratory syndrome coronavirus receptor for cellular entry. Proceedings of the National Academy of Sciences, 2005. 102(22): p. 7988-7993.

13. Zhou, P., et al., A pneumonia outbreak associated with a new coronavirus of probable bat origin. Nature, 2020.

14. Chen, Y., et al., Structure analysis of the receptor binding of 2019-nCoV. Biochemical and Biophysical Research Communications, 2020.

15. $\mathrm{Xu}, \mathrm{X}$., et al., Evolution of the novel coronavirus from the ongoing Wuhan outbreak and modeling of its spike protein for risk of human transmission. Science China Life Sciences, 2020.

16. Xin Zou, K.C., Jiawei Zou, Peiyi Han, Jie Hao, Zeguang Han, The single-cell RNA-seq data analysis on the receptor ACE2 expression reveals the potential risk of different human organs vulnerable to Wuhan 2019-nCoV infection. Front. Med.: p. 0-.

17. Zhao, Y., et al., Single-cell RNA expression profiling of ACE2, the putative receptor of Wuhan 2019nCov. bioRxiv, 2020: p. 2020.01.26.919985.

18. Zhang, H., et al., The digestive system is a potential route of 2019-nCov infection: a bioinformatics analysis based on single-cell transcriptomes. bioRxiv, 2020: p. 2020.01.30.927806.

19. Jia, H.P., et al., ACE2 Receptor Expression and Severe Acute Respiratory Syndrome Coronavirus Infection Depend on Differentiation of Human Airway Epithelia. Journal of Virology, 2005. 79(23): p. 14614.

20. Chai, X., et al., Specific ACE2 Expression in Cholangiocytes May Cause Liver Damage After 2019nCoV Infection. bioRxiv, 2020: p. 2020.02.03.931766.

21. Hamming, I., et al., Tissue distribution of ACE2 protein, the functional receptor for SARS coronavirus. A first step in understanding SARS pathogenesis. The Journal of Pathology, 2004. 203(2): p. 631-637.

22. $\mathrm{Xu}, \mathrm{H}$., et al., High expression of ACE2 receptor of 2019-nCoV on the epithelial cells of oral mucosa. International Journal of Oral Science, 2020. 12(1): p. 8. 
23. Donoghue, M., et al., A novel angiotensin-converting enzyme-related carboxypeptidase (ACE2) converts angiotensin I to angiotensin 1-9. Circ Res, 2000. 87(5): p. E1-9.

24. Patel, V.B., et al., Role of the ACE2/Angiotensin 1\&\#x2013;7 Axis of the Renin\&\#x2013;Angiotensin System in Heart Failure. Circulation Research, 2016. 118(8): p. 1313-1326.

25. Haga, S., et al., Modulation of TNF-alpha-converting enzyme by the spike protein of SARS-CoV and ACE2 induces TNF-alpha production and facilitates viral entry. Proc Natl Acad Sci U S A, 2008. 105(22): p. 7809-14.

26. Lambert, D.W., et al., Tumor necrosis factor-alpha convertase (ADAM17) mediates regulated ectodomain shedding of the severe-acute respiratory syndrome-coronavirus (SARS-CoV) receptor, angiotensin-converting enzyme-2 (ACE2). J Biol Chem, 2005. 280(34): p. 30113-9.

27. Lambert, D.W., et al., Calmodulin interacts with angiotensin-converting enzyme-2 (ACE2) and inhibits shedding of its ectodomain. FEBS Lett, 2008. 582(2): p. 385-90.

28. Haga, S., et al., TACE antagonists blocking ACE2 shedding caused by the spike protein of SARS-CoV are candidate antiviral compounds. Antiviral Res, 2010. 85(3): p. 551-5.

29. Heurich, A., et al., TMPRSS2 and ADAM17 cleave ACE2 differentially and only proteolysis by TMPRSS2 augments entry driven by the severe acute respiratory syndrome coronavirus spike protein. J Virol, 2014. 88(2): p. 1293-307.

30. Simmons, G., et al., Inhibitors of cathepsin L prevent severe acute respiratory syndrome coronavirus entry. Proc Natl Acad Sci U S A, 2005. 102(33): p. 11876-81.

31. Jia, H.P., et al., Ectodomain shedding of angiotensin converting enzyme 2 in human airway epithelia. Am J Physiol Lung Cell Mol Physiol, 2009. 297(1): p. L84-96.

32. Kuba, K., et al., A crucial role of angiotensin converting enzyme 2 (ACE2) in SARS coronavirusinduced lung injury. Nature Medicine, 2005. 11(8): p. 875-879.

33. Channappanavar, R., et al., Sex-Based Differences in Susceptibility to Severe Acute Respiratory Syndrome Coronavirus Infection. Journal of immunology (Baltimore, Md. : 1950), 2017. 198(10): p. 4046-4053.

34. Luo, Y., et al., Association of ACE2 genetic polymorphisms withhypertension-related target organ damages in south Xinjiang. Hypertension Research, 2019. 42(5): p. 681-689.

35. Renieri, A., et al., ACE2 variants underlie interindividual variability and susceptibility to COVID-19 in Italian population. 2020: p. 2020.04.03.20047977.

36. Kleine-Weber, $\mathrm{H}_{\text {., }}$ et al., Polymorphisms in dipeptidyl peptidase 4 reduce host cell entry of Middle East respiratory syndrome coronavirus. Emerg Microbes Infect, 2020. 9(1): p. 155-168.

37. Pinto, B.G., et al., ACE2 Expression is Increased in the Lungs of Patients with Comorbidities Associated with Severe COVID-19. medRxiv, 2020: p. 2020.03.21.20040261.

38. Leung, J.M., et al., ACE-2 Expression in the Small Airway Epithelia of Smokers and COPD Patients: Implications for COVID-19. medRxiv, 2020: p. 2020.03.18.20038455.

39. Zhu, Z., et al., Potent cross-reactive neutralization of SARS coronavirus isolates by human monoclonal antibodies. Proc Natl Acad Sci U S A, 2007. 104(29): p. 12123-8.

40. Chen, L., et al., Convalescent plasma as a potential therapy for COVID-19. Lancet Inf Dis, 2020.

41. Al-Qahtani, A.A., et al., Middle east respiratory syndrome corona virus spike glycoprotein suppresses macrophage responses via DPP4-mediated induction of IRAK-M and PPARgamma. Oncotarget, 2017. 8(6): p. 9053-9066.

42. Liu, J., et al., Hydroxychloroquine, a less toxic derivative of chloroquine, is effective in inhibiting SARS-CoV-2 infection in vitro. Cell Discovery, 2020. 6(1): p. 16.

43. Chen, X., et al., Detectable serum SARS-CoV-2 viral load (RNAaemia) is closely associated with drastically elevated interleukin 6 (IL-6) level in critically ill COVID-19 patients. medRxiv, 2020: $\mathrm{p}$. 2020.02.29.20029520.

44. Imai, Y., et al., Angiotensin-converting enzyme 2 protects from severe acute lung failure. Nature, 2005. 436(7047): p. 112-116. 
45. Zou, Z., et al., Angiotensin-converting enzyme 2 protects from lethal avian influenza A H5N1 infections. Nature Communications, 2014. 5(1): p. 3594.

46. Yang, P., et al., Angiotensin-converting enzyme 2 (ACE2) mediates influenza H7N9 virus-induced acute lung injury. Scientific Reports, 2014. 4(1): p. 7027.

47. Ishiyama, Y., et al., Upregulation of angiotensin-converting enzyme 2 after myocardial infarction by blockade of angiotensin II receptors. Hypertension, 2004. 43(5): p. 970-6.

48. Ferrario, C., J. Jessup, and M. Chappell, Effect of angiotensin-converting enzyme inhibition and angiotensin II receptor blockers on cardiac angiotensin-converting enzyme 2. Circulation 2005. 111: p. 2605-10.

49. Klimas, J., et al., Perinatally administered losartan augments renal ACE2 expression but not cardiac or renal Mas receptor in spontaneously hypertensive rats. J Cell Mol Med, 2015. 19(8): p. 1965-74.

50. Furuhashi, M., et al., Urinary angiotensin-converting enzyme 2 in hypertensive patients may be increased by olmesartan, an angiotensin II receptor blocker. Am J Hypertens, 2015. 28(1): p. 15-21.

51. Gurwtiz, D., Angiotensin Receptor Blockers as Tentative SARS-CoV-2 Therapeutics. Drug Dev Res, 2020.

52. Di Guardo, G., SARS-CoV-2, hypertension and ACE inhibitors. Br Med J, 2020. 368.

53. Sriram, K. and P.A. Insel, Dangers of ACE inhibitor and ARB usage in COVID-19: evaluating the evidence. 2020: p. 2020.03.25.20043927.

54. Lu, R., et al., Genomic characterisation and epidemiology of 2019 novel coronavirus: implications for virus origins and receptor binding. The Lancet, 2020. 395(10224): p. 565-574.

55. Yang, G., et al., Angiotensin II Receptor Blockers and Angiotensin-Converting Enzyme Inhibitors Usage is Associated with Improved Inflammatory Status and Clinical Outcomes in COVID-19 Patients With Hypertension. 2020: p. 2020.03.31.20038935.

56. Feng, Z., et al., The Use of Adjuvant Therapy in Preventing Progression to Severe Pneumonia in Patients with Coronavirus Disease 2019: A Multicenter Data Analysis. 2020: p. 2020.04.08.20057539.

57. Bean, D., et al., Treatment with ACE-inhibitors is associated with less severe disease with SARSCovid-19 infection in a multi-site UK acute Hospital Trust. 2020: p. 2020.04.07.20056788.

58. Zeng, Z., et al., Hypertension in patients hospitalized with COVID-19 in Wuhan, China: A singlecenter retrospective observational study. 2020: p. 2020.04.06.20054825.

59. Huentelman, M.J., et al., Structure-based discovery of a novel angiotensin-converting enzyme 2 inhibitor. Hypertension, 2004. 44(6): p. 903-6.

60. Turner, A.J., J.A. Hiscox, and N.M. Hooper, ACE2: from vasopeptidase to SARS virus receptor. Trends Pharmacol Sci, 2004. 25(6): p. 291-4.

61. Michel, N., et al., The Nef protein of human immunodeficiency virus establishes superinfection immunity by a dual strategy to downregulate cell-surface CCR5 and CD4. Curr Biol, 2005. 15(8): p. 714-23.

62. Yu, C.M., et al., Cardiovascular complications of severe acute respiratory syndrome. Postgraduate medical journal, 2006. 82(964): p. 140-144.

63. Khan, A., et al., A pilot clinical trial of recombinant human angiotensin-converting enzyme 2 in acute respiratory distress syndrome. Critical Care, 2017. 21(1): p. 234.

64. Sun, M.L., et al., [Inhibitors of RAS Might Be a Good Choice for the Therapy of COVID-19 Pneumonia]. Zhonghua Jie He He Hu Xi Za Zhi, 2020. 43(0): p. E014. 